Vol. 25, No. 01, pp.09-17/Junio 2012

\title{
Diagnóstico de Procesos Previos a la Aplicación de la Manufactura Esbelta
}

\author{
Y. Juárez López ${ }^{1}$, A. Pérez Rojas ${ }^{1}$ y J. Rojas Ramírez ${ }^{2 *}$ \\ ${ }^{1}$ Centro de Investigación Avanzada en Ingeniería Industrial, \\ Universidad Autónoma del Estado de Hidalgo \\ Pachuca, Hidalgo, México \\ e-mail: yolandaj@uaeh.edu.mx , auropr@uaeh.edu.mx \\ ${ }^{2}$ Escuela Superior de Ingeniería Mecánica y Eléctrica, \\ Instituto Politécnico Nacional \\ Zacatenco D. F., México \\ e-mail: jrojasr@ipn.mx
}

(Recibido/received: 06-Septiembre-2011; aceptado/accepted: 26-Febrero-2012)

\section{RESUMEN}

Una etapa necesaria para la formulación de una guía de solución a problemas de satisfacción de la demanda es el diagnóstico previo. En este artículo se describe la manera en que éste se llevó cabo para el caso de una empresa de material ferroviario en el Estado de Hidalgo, México. Las fases que se desarrollan son: la descripción del sistema bajo estudio, la observación y la evaluación del desempeño del sistema, la puntualización de los problemas detectados y la asociación de éstos con las causas que los originan. Como criterio para la evaluación del desempeño se ha seleccionado el paradigma de la manufactura esbelta, de raíces japonesas, considerado el más adecuado al caso en razón de atacar los desperdicios del proceso y por su carácter de solucionar participativamente. En este caso los desperdicios por atacar son los largos tiempos de preparación, la elevada tasa de productos no conformes y la falta de mantenimiento al equipo.

Palabras clave: Diagnóstico, Manufactura Esbelta, Desperdicios, Empresas de Transporte Ferroviario

\begin{abstract}
In implementing a solution guide for manufacturing problems such as an unsatisfied demand, a previous diagnosis is necessary. In this paper we describe how this was developed for the case of railroad material enterprises at the Mexican State of Hidalgo. The phases considered are: the description of the system under study, the capture and assess of the system performance, the definition of the observed problems, and its association with the causes generating them. As the performance evaluating criteria the Lean Production paradigm, originated in Japan, has been selected, due to its adequacy to the case because of its focus in process waste reduction and participative problem solving. For this case, the wastes to cope with are the long set-up times, the high rate of bad products, and the lack of adequate equipment maintenance.
\end{abstract}

Keywords: Diagnosis, Lean Manufacture, Railroad Material Enterprises, Wastes

\footnotetext{
* Autor para la correspondencia
} 


\section{INTRODUCCIÓN}

El diagnóstico que se presenta es una fase previa dentro de un proyecto en desarrollo, que está orientado a aportar elementos de mejora a los procesos de producción de empresas de manufactura de medios de transporte ferroviario (EMMTF), ubicadas en Ciudad Sahagún, Estado de Hidalgo, México. La razón del diagnóstico es preparar una fase posterior de propuesta de los elementos de mejora dentro de una guía de aplicación al caso. En este artículo se precisa cómo el diagnóstico de los procesos permite: identificar los problemas que se presentan, compararlos con los lineamientos que señala la Manufactura Esbelta (ME) y relacionarlos con las causas que los generan.

Como antecedente, se ha puesto de manifiesto la importancia que reviste la propuesta de lograr que las EMMTF estudiadas alcancen niveles de desempeño equiparables con los más altos del contexto globalizado, para que sus productos paulatinamente sean más competitivos.

Se ha realizado la revisión de métodos de la ME viables para la línea de fabricación estudiada, todos ellos dirigidos a la eliminación de los desperdicios vinculados a estos procesos. Para conseguir la guía es necesario describir el aspecto técnico de la línea de fabricación, conocer la terminología utilizada y comprender la complejidad y la interacción entre sus etapas. Las fuentes de información utilizadas han sido documentos técnicos de las constructoras de unidades ferroviarias, así como el conocimiento directo por visitas y entrevistas a personal de las EMMTF.

Se ha propuesto la Manufactura Esbelta como el paradigma para sustentar esta propuesta, porque la fabricación de vagones ferroviarios está caracterizada por múltiples bloqueos. Los procesos de la línea de fabricación son interesantes de estudiar, en razón de presentar una gran cantidad de desperdicios, creados por el número de piezas innecesarias, el de defectos generados, el de retrabajos requeridos y los incumplimientos en entregas. Como verificación de esta elección, se lleva a cabo una corrida comparativa en un simulador de procesos.

Dentro de la línea de fabricación, los productos se conforman a lo largo de las etapas de montaje metálico, pintura y vestidura. A su vez, la de pintura contempla cinco grandes procesos. Éstos son los de limpieza por granalla, primario de pintura, plaste, pintura intermedia y pintura final. La observación de que en ellos hay una alta generación de desperdicios es la razón de elegirlos para la aplicación del diagnóstico. A cada uno de estos desperdicios se asocia las causas principales, con lo que se facilita la selección de los métodos de la ME de mayor factibilidad para ser empleados en la solución.

Desde un punto de vista científico, el diagnóstico como detector de la situación actual de las EMMTF ha fungido como facilitador en la búsqueda de soluciones esbeltas, como se pone de manifiesto en este trabajo. El mismo consta de una descripción del sistema objeto de estudio, el análisis del desempeño actual, la caracterización de los problemas y, finalmente, la ubicación de las causas por combatir.

En general la propuesta de esta investigación busca superar la toma de decisiones improvisada para la mejora de la industria por una formal, en la que se seleccione la mejor alternativa por la comparación basada en las causas ciertas de los problemas, tal como lo hace el pensamiento esbelto.

La aportación técnica se concreta, sobre el sustento anterior, en un modelo de aplicación para guiar eficazmente las acciones de mejora en los procesos estudiados, con garantía de solucionar los problemas de fondo y alcanzar desempeños más elevados.

\section{METODOLOGÍA}

\section{Análisis de la producción de material ferroviario}

En general, cuando se diseñan los procesos productivos, el objetivo principal que se quiere lograr es que los productos terminados estén conformes a las especificaciones planteadas (Sipper, et al., 1998). Sin embargo, en los procesos que generan productos intermedios aparecen problemas de desperdicios al no cumplirse con las especificaciones que éstos requieren para continuar la línea de fabricación, provocando retoques y retrabajos, utilizando más horas de las necesarias, almacenamientos excesivos, etcétera (Schonberger, 1987).

Entre las EMMTF consideradas para este estudio es posible identificar elementos comunes en el diagnóstico de sus procesos, paso previo para la aplicación de la ME.

En general, cada uno de los procesos estudiados se conforma por: 
- La recepción de lotes de materiales y productos intermedios $y$ su correspondiente almacenamiento,

- La ejecución de actividades de preparación en productos, máquinas y dispositivos empleados en el proceso,

- El proceso principal, en el que efectivamente se lleva a cabo una transformación del producto,

- La ejecución de actividades de terminación del proceso,

- Las inspecciones y los almacenamientos intermedios entre estaciones de trabajo, y

- La inspección final y el almacenamiento del producto terminado.

En todos ellos la utilización de personal se efectúa en los turnos normal y de fin de semana. Las jornadas de lunes a viernes se destinan a los productos sin defecto $\mathrm{y}$ sábados y domingos a la recuperación de las unidades que fueron rechazadas en la inspección.

Los recursos de transformación presentan periodos de indisponibilidad por fallas, estos se evalúan mediante la medición del tiempo promedio entre fallas $\mathrm{y}$, al ser corregidas por el mantenimiento, se estima el tiempo promedio para reparar. La eficiencia del mantenimiento incide en estos dos indicadores.

En el entorno mexicano, para las EMMTF de Ciudad Sahagún se fabrican diversos tipos de unidades ferroviarias para el transporte de pasajeros o de carga. La descripción de las actividades que constituyen la línea de fabricación inicia a continuación.

\section{Etapa de montaje metálico}

Para la etapa de montaje metálico, desde un almacén de materia prima se pasa a los talleres de fabricación metálica pesada y ligera, para su transformación a piezas del producto y posteriormente accede al almacén de partes acabadas. Éstas son llevadas después a la línea de subensamble de las partes. La estructura de la caja metálica está constituida por: bastidor, frentes, costados y techo. El producto semiacabado es llevado a la nave de montaje, en donde se efectúa una serie de operaciones de soldadura, enderezado, colocación de soportes, lámina, pruebas de hermeticidad y hojalateado.

Además se efectúan las operaciones de preparación, aplicación de material antirruido, colocación de pisos y colocación de ventanas y puertas. A través de soldadura, se realiza la unión de los subensambles, hasta completar la estructura metálica de la caja del vagón.

Etapa de pintura y sus procesos

Una vez realizado lo anterior, la caja metálica pasa a la nave de pintura en donde se le practica la serie de operaciones mencionada, que incluye lijados, pulidos y la aplicación de pinturas, para realzar la presentación del producto final. Esquemáticamente se muestra la línea de pintura en la Fig. 1.

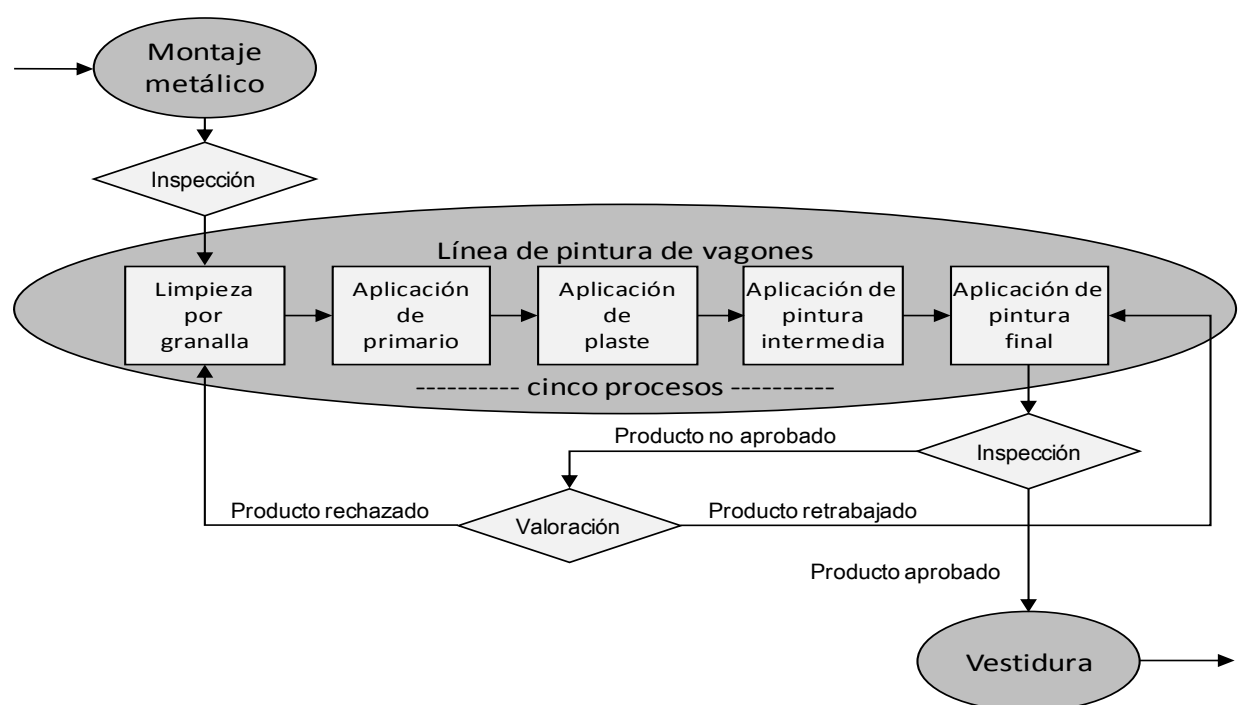

Fig. 1. Línea de pintura de vagones analizada y sus cinco procesos.

Vol. 25, No. 01, pp. 09-17/Junio 2012 
A esta etapa la conforman los procesos de limpieza por granalla, aplicación de primario de pintura, aplicación de plaste, aplicación de pintura intermedia y aplicación de pintura final, que a continuación se detallan.

- Proceso de limpieza por granalla: se utiliza para decapar las grandes superficies de la caja metálica, eliminando el óxido y las imperfecciones del laminado y preparando el material para su pintado. Posteriormente se inspecciona por control de calidad;

- Proceso de aplicación de primario de pintura: se protege al metal del óxido y se evita la corrosión futura y es aplicada inclusive sobre partes ocultas;

- Proceso de aplicación de plaste: este material tiene como finalidad cubrir las deformaciones presentadas en la estructura metálica, y luego es lijado para quitar el exceso, el brillo e imperfecciones en el mismo y también para verificar la presencia de poros;

- Proceso de aplicación de pintura intermedia: tiene como finalidad cubrir las fallas manifestadas en la etapa anterior y también como fondo para la aplicación de la pintura siguiente;

- Proceso de aplicación de pintura final: después de realizar una limpieza con solvente especial, se aplica esta pintura en la finalización de la etapa y entonces se presenta a control de calidad para su inspección.

Para cada uno de los procesos se distinguen tres componentes, que son las actividades de preparación o alistamiento (tiempo anterior), de aplicación efectiva del paso de transformación (tiempo principal) y de terminación (tiempo posterior). Como ejemplo de estos pasos, la aplicación de la pintura intermedia es precedida por la limpieza con solvente de la unidad ferroviaria y el recubrimiento de puertas, ventanas y conexiones eléctricas y es terminada con el retiro de las protecciones de las partes cubiertas, con el conocimiento del tiempo promedio que toma cada uno de los subprocesos.

De la actividad de inspección del producto que finaliza la etapa de pintura pueden suceder tres diferentes situaciones, a saber:

- Si es aprobado, pasa a la siguiente etapa

- Si es rechazado, se queda en espera de dos casos posibles:
- Inconformidad leve, recuperable por retrabajo, es devuelto al proceso de pintura final,

- Inconformidad grave, es regresado al proceso de granalla para ser tratado igual que la caja metálica de nuevo ingreso a la etapa de pintura.

\section{Etapa de vestidura}

En la etapa de vestidura las principales operaciones son: colocación de equipo eléctrico, instalación de parabrisas, montaje de equipo neumático, instalación de grupo motor, cableado de ventilación, revestimiento de paneles y colocación de paneles en plafón.

El producto en proceso vuelve a la nave de pintura para la aplicación de un esmalte final, así como la colocación de insignias, de pasamanos y de asientos. Fuera de la nave se procede al montaje de las estructuras rodantes, o bogies, para realizar pruebas estáticas y dinámicas y, de no encontrar problemas, se lleva el vagón al almacén de producto terminado.

El producto liberado para su embarque se protege con lonas y material plástico para evitar daños como rayones, suciedad, engrasado, salpicaduras y polvo. Una vez empacado, se monta el producto en las plataformas para su distribución y su traslado al lugar de destino. Mientras, permanece en los almacenes de producto terminado.

\section{Evaluación del desempeño de acuerdo a los métodos esbeltos}

El planteamiento de aplicar métodos japoneses, como los que integran el paradigma de la manufactura esbelta, en cierto grado deja la incógnita de su efectividad en un entorno geográfico diferente (Coriat, 1995). Se hace notar que en su país de origen, se han publicado resultados de las experiencias desde el año 1977 (New, 2007). El caso de su aplicación en América del Norte, en particular en la industria mexicana del automóvil es abordado por Juárez Núñez y Babson (1999).

Bednarek y Niño Luna (2008) abordan cuestiones de la implantación de la manufactura esbelta en empresas medianas y pequeñas de México y realizan la propuesta de un modelo de cuatro niveles progresivos, en el que señalan como partes importantes la inclusión de normas ISO, kaizen, tecnología de grupos, teoría de las restricciones, TPM y SMED. 
Otros autores que se han dedicado a aplicaciones de la ME en contextos fuera del Japón, y que han generado literatura en español con la explicación de los diferentes métodos que la conforman, son Lefcovich (2009), Galgano (2006) y Villaseñor y Galindo (2008).

En un estudio de Nakamura y colaboradores (1998) el interés se ha dirigido a recoger la evidencia empírica de las mejoras en diversas medidas de desempeño, como el porcentaje de piezas correctas o de los tiempos de paro, comparativamente entre empresas americanas y japonesas, encontrando los contrastes por la implantación de métodos esbeltos.

Con el fin de asociar cada uno de los problemas anteriores con el método de la manufactura esbelta esbelto que tiene mayor relación con el fenómeno particular que lo produce, se llega a la selección de aquellos con mayor factibilidad de aplicación, que se indican a continuación.

- Jidoka y poka-yoke: la utilización de mecanismos de detección y prevención de operaciones erróneas corresponden a la solución de un alto número de productos no conformes (Hirano, 1992) (Suzaki, 1993);

- Mantenimiento Productivo Total (TPM): con su aplicación se busca mejorar lo preventivo y lo correctivo, al alargar el tiempo entre apariciones de falla y reducir el tiempo de las intervenciones (Allen, et al., 2001);

- Soikufu: la aportación de ideas por los trabajadores para mejorar la eficiencia de las operaciones se observa como la posibilidad de uniformizar los tiempos de proceso, a través de la reducción de la variabilidad de los mismos (Domínguez Machuca, et al., 1995);

- Shojinka: su aplicación sirve para agilizar los movimientos de material en la planta al reducir las distancias entre estaciones, generar trayectorias más directas y lograr una mejor visualización de las estaciones anterior y posterior (Monden, 1990);

- Reducción de tiempos de preparación (SMED): las esperas por tiempos de preparación son abordadas por este método al promover su reducción a menos de 10 minutos (Béranguer, 1994);

- Otras suplementarias: para aprovechar el pensamiento esbelto de manera integral es conveniente iniciar la solución con las Cinco Eses para establecer el clima de cambio y los métodos de mapeo del flujo de valor (VSM) para ubicar los procesos de mayor desperdicio, así como culminar con la visión de la mejora continua que enseña kaizen, para que los cambios sean efectivos y permanentes (Schonberger, 1996).

Con esta guía, lo procedente a continuación es la asignación de valores detallados a los parámetros que se localizan en el origen de los problemas, para cuantificar la contribución de cada uno, junto con la determinación de los valores objetivo para su modificación.

La estructuración de un procedimiento más concreto de solución, para no quedar en un listado de métodos, da lugar al modelo de manufactura esbelta que constituye la fase siguiente del proyecto de investigación. En ella se emplea un simulador de procesos, para llevar un conjunto de experimentos, que permitan asegurar su correcta operatividad.

\section{Focalización de los problemas observados y sus causas}

En esta sección se procede a expresar en términos estadísticos el estado de la problemática observada en el caso de estudio.

El análisis resultante del diagnóstico de estos procesos productivos arroja una alta frecuencia de rechazo a la salida de la etapa de pintura final. En la Tabla 1 se presentan los resultados promedio de una semana típica de labores de la línea de fabricación, al ser inspeccionado el producto a la salida de la etapa de pintura, con sólo $60 \%$ de producto aprobado a la primera.

Puesto que las cifras pueden parecer excesivas se recurrió a consultar otras fuentes, que corroboran los órdenes de magnitud. En la pintura final de automóviles se reporta el 65\%, como límite mínimo (Ulgen, et al., 1998) y en el control de calidad de pintura final de autobuses se cita el 75\% (Dengiz, et al., 2007).

Tabla 1. Resultados de la inspección en pintura

\begin{tabular}{lc}
\hline Resultado de la inspección & Porcentaje \\
\hline Aprobado & $60 \%$ \\
$\begin{array}{l}\text { Inconformidad leve (recuperable } \\
\text { con retrabajo) }\end{array}$ & $28 \%$ \\
$\begin{array}{l}\text { Inconformidad grave (vuelve a } \\
\text { granalla al inicio de la etapa) }\end{array}$ & $12 \%$ \\
\hline
\end{tabular}

Otros resultados obtenidos del diagnóstico reflejan desempeños no deseables en varios aspectos, de los 
cuales los principales son: sobreproducción e inventarios, tiempo extra y esfuerzos perdidos. Todo lo anterior se puede relacionar con desperdicios, es decir, actividades que no aportan valor agregado al producto, pero que consumen recursos, aspecto que coincide con las causas que se dedica a combatir la manufactura esbelta (Fogarty, et al., 1991) (Womack, 1991).

En los procesos estudiados los aspectos problemáticos más sobresalientes son:

- La baja tasa de productos aprobados en la inspección a la primera;

- Los tiempos de paro de equipo y maquinaria por un mantenimiento incorrecto;

- La generación de inventarios intermedios excesivos entre etapas por la variabilidad de los tiempos de proceso y la carencia de un balance adecuado;

- Los movimientos innecesarios de material y personal en una deficiente distribución en planta;

- Las esperas por tiempos de preparación al iniciar cada unidad de producto.

Con la finalidad de concretar la medición del desempeño, se recurrió a tres indicadores. Con ellos se pueden probar los resultados de las alternativas de solución. Los tres puntos son: la disponibilidad (D), la entrega a tiempo (T) y la calidad (Q). Se promueve que sean indicadores normalizados, en escalas de 0 a 1 , para tener la facilidad posterior de ser combinados.

El índice D, disponibilidad, es medido en el proceso de pintura final. Se obtiene dividiendo el tiempo en el cual la estación de trabajo no se encuentra disponible (por encontrarse en reparación tras el efecto de una falla) entre el tiempo total (en este caso de 5 días) y restando el resultado de uno.

El índice T, de entregas puntuales, se relaciona con el tiempo real del fin de la producción comparado con el ofrecido al cliente. Todo retraso en la entrega del producto terminado reduce proporcionalmente el valor del mismo, que es de 1 si hubo cumplimiento.

Por su parte, el índice de calidad Q es la proporción de productos aceptados a la primera, sin considerar los retrabajos ni los rechazos, en forma de cociente.

\section{Prueba y validación de un modelo de simulación}

Se ha recurrido a un simulador de procesos con la construcción de un modelo de la línea de pintura en el paquete de software ProModel, de acuerdo con la estructura descrita y con los datos del caso. Se cotejó el comportamiento del modelo contra el del sistema real y así se valida su representatividad.

A raíz de esta descripción se han puntualizado datos adicionales, necesarios para el simulador, y que permiten una mayor comprensión del proceso. En términos estadísticos, la descripción de la línea presentada anteriormente se relaciona con los tiempos en minutos señalados en la Tabla 2. Se detallan los tiempos de los subprocesos anterior, principal y posterior, pertenecientes a la etapa de pintura. Se indican los valores promedio y se asumen distribuciones normales.

Tabla 2. Tiempos de Proceso en la línea (min.)

\begin{tabular}{lccc}
\hline $\begin{array}{l}\text { Proceso de } \\
\text { pintura }\end{array}$ & $\begin{array}{c}\text { Tiempo } \\
\text { anterior }\end{array}$ & $\begin{array}{c}\text { Tiempo } \\
\text { principal }\end{array}$ & $\begin{array}{c}\text { Tiempo } \\
\text { posterior }\end{array}$ \\
\hline Granalla & 59 & 355 & - \\
Primario & 125 & 238 & 73 \\
Plaste & 239 & 117 & 70 \\
Intermedia & 210 & 151 & 66 \\
Final & 151 & 198 & 63 \\
\hline
\end{tabular}

Por su parte, la Tabla 3 muestra los indicadores del mantenimiento por proceso de pintura, en cuanto al preventivo, por el tiempo medio transcurrido entre dos fallas consecutivas, y en cuanto al correctivo, por la duración de la intervención, en el tiempo medio para reparar.

Tabla 3. Tiempos de mantenimiento (min.)

\begin{tabular}{lcc}
\hline $\begin{array}{l}\text { Proceso de } \\
\text { pintura }\end{array}$ & $\begin{array}{c}\text { Tiempo medio } \\
\text { entre fallas }\end{array}$ & $\begin{array}{c}\text { Tiempo medio } \\
\text { para reparar }\end{array}$ \\
\hline Granalla & 785 & 142 \\
Primario & 785 & 135 \\
Plaste & 785 & 118 \\
Intermedia & 785 & 135 \\
Final & 785 & 155 \\
\hline
\end{tabular}

En seguida se realiza una corrida para obtener el desempeño de la línea. Los índices obtenidos son: $\mathrm{D}=0.9134, \mathrm{~T}=0.5198$ y $\mathrm{Q}=0.6178$. Estos datos se consideran inaceptables. Representan el comportamiento del estado inicial del objeto de estudio y, dado su rango potencial entre 0 y 1 , se justifica llevar a cabo las mejoras. 
Después de la corrida del modelo de simulación del sistema original, se ejecutan otras dos corridas con las posibilidades de mejora. Estas son la solución tradicional que el sistema emplearía y la que propone la manufactura esbelta.

\section{Solución con un esquema tradicional}

Con la solución tradicional, el flujo es reforzado mediante la redundancia de las funciones básicas, el desacoplamiento de los desempeños individuales mediante almacenes temporales, la especialización de personal, manejo de materiales y otras funciones como el mantenimiento.

Para la línea de pintura, la aplicación de los principios de este enfoque procede con la protección mediante inventarios, así que los almacenes de proceso aumentan en capacidad a 10 unidades. En previsión de la ausencia de operarios, materiales o dispositivos, aumenta su número. Así, los operarios de lunes a viernes pasan de 5 a 6 , los de fin de semana 3 a 4 y las llegadas de materia prima en $10 \%$ de más. Las modificaciones en la calidad de la pintura final permiten cambiar los porcentajes originales a $66 \%$ de producto correcto, $31 \%$ de retrabajos y $3 \%$ de rechazos.

\section{Solución con manufactura esbelta}

Con el enfoque de la manufactura esbelta como guía de solución, se centra la atención en la eliminación de desperdicios. Para ello, se reducen la variabilidad de los procesos, los inventarios y los tamaños de los lotes. Consecuentemente, la media y la desviación estándar de los tiempos principales bajan en $10 \%$ y, en una medida similar, los de los tiempos de preparación y traslado. Igualmente, se promueve la calidad del proceso para obtener las mismas proporciones citadas de $66 \%, 31 \%$ y $3 \%$ respectivamente.

\section{Comparación de resultados de la simulación}

Se experimentan los dos cambios hechos sobre el modelo original. Por cada experimento se realizan 50 réplicas en la simulación, para fines de representatividad. En la Tabla 4 se reportan los tres índices de medición del desempeño del sistema ya explicados.

Tabla 4. Resultados de la simulación preliminar

\begin{tabular}{llll}
\hline Paradigma de & Disponi- & Entrega a & Calidad \\
\hline
\end{tabular}

\begin{tabular}{llll}
\hline solución & bilidad & tiempo & \\
\hline Original & 0.9134 & 0.5198 & 0.6178 \\
Tradicional & 0.8629 & 0.6294 & 0.6832 \\
Esbelto & 0.9123 & 0.6372 & 0.6901 \\
\hline
\end{tabular}

Con estos resultados, considerados preliminares puesto que los métodos de la manufactura esbelta tienen mucho más potencial que el aquí mostrado, en esta etapa permiten sustentar lo adecuado del diagnóstico descrito.

Por una parte, se refuerza lo necesario que es modificar el proceso original, porque sus indicadores no son satisfactorios. En segundo término, se muestra que la manufactura esbelta, en lugar de la solución que tradicionalmente se aplicaría, conduce a una mejora más sustancial, al orientarse a la reducción de los desperdicios de los procesos. Adicionalmente, la etapa de mejora continua, característica del enfoque esbelto, promovería la observación constante de nuevas oportunidades de elevar el desempeño del sistema.

\section{CONCLUSIONES}

La adopción de la Manufactura Esbelta como paradigma de solución al problema de incumplimientos en la entrega de productos en la línea de fabricación de material ferroviario estudiada ha permitido dilucidar el procedimiento de solución.

Después de ubicar a la etapa de diagnóstico como el antecedente a la estructuración de una propuesta formal a manera de guía para la empresa, se ha desagregado en cuatro pasos. Estos son: la recolección de información del caso de estudio, la evaluación de la situación conforme a un criterio seleccionado, la puntualización del problema por sus efectos y la asociación de los mismos con las causas que se habrán de combatir.

La información recolectada del caso particular ha mostrado las particularidades de la manufactura de material ferroviario en empresas del Estado de Hidalgo, en México.

Se recurrió a un modelo de simulación en computadora que, además de forzar a obtener valores estadísticos a las variables del proceso, tiene la ventaja de entregar valores numéricos para puntualizar los índices de desempeño.

La evaluación se ha conformado al pensamiento esbelto, que pone el énfasis en detectar y reducir los desperdicios, tanto de acumulaciones y traslados innecesarios, como de paros y esperas y de calidad no 
conforme. La selección de este paradigma de origen japonés se ha basado en su mejor adecuación al caso mexicano, al contrario de alternativas que tradicionalmente se seguirían, como la automatización, la redundancia de recursos, la especialización de la mano de obra o programas de calidad.

Bajo esta visión, los problemas diagnosticados para el caso particular se han concretado en los largos tiempos de preparación para los procesos de pintura de primario, intermedia y final, la alta tasa de producto no aprobado, tanto por retrabajo como por rechazo y las indisponibilidades por falla del equipo, ya que todo ello incide en el cumplimiento a tiempo con el cliente.

Consecuentemente, el trabajo futuro dentro del proyecto está orientado a la propuesta de una guía ordenada de acciones que combatan las causas de los problemas. Con ello se anticipa que se adoptarán métodos de extracción japonesa tales como SMED para lograr tiempos de preparación más bajos, poka-yoke para instrumentar ideas y dispositivos contra fallas de calidad y mantenimiento productivo total para el aumento de la disponibilidad del equipo. Más adelante, la implantación de la guía validará la propuesta, complementada con el enfoque kaizen, o de la mejora continua. Su herramienta de soporte será, como en el caso ilustrado, el simulador de procesos.

\section{REFERENCIAS}

Allen, J., C. Robinson \& D. Stewart (ed.) (2001). Lean Manufacturing: A Plant Floor Guide, Dearborn MI: Soc. Manufacturing Engineers.

Bednarek, M. \& L. F. Niño Luna (2008). The Selected Problems of Lean Manufacturing Implementation in Mexican SMEs, en Lean Business Systems and Beyond, T. Koch (ed.), Vol. 257, pp. 239-247, Boston: Springer.

Béranger, P. (1994). En Busca de la Excelencia Industrial: Just in-Time: Las Nuevas Reglas de la Producción, México: Limusa Noriega.

Coriat, B. (1995). Pensar al Revés: Trabajo y Organización en la Empresa Japonesa, 2a ed., México: Siglo Veintiuno.

Dengiz, B. \& O. Belgin (2007). Paintshop Production Line Optimization using Response Surface Methodology, en Proceedings of the 2007 Winter Simulation Conference, S. G. Henderson, et al. (ed.), pp. 1667-1672.
Domínguez Machuca, J. A., et al. (1995). Dirección de Operaciones: Aspectos Tácticos y Operativos en la Producción y los Servicios, Madrid: McGraw-Hill.

Fogarty, D. W., J. H. Blackstone Jr. \& T. R. Hoffmann (1991). Production \& Inventory Management, 2a ed., Cincinnati OH: South-Western.

Galgano, A. (2006). Las Tres Revoluciones: Caza del Desperdicio. Doblar la Productividad con Lean Production, Madrid: Díaz de Santos.

Hirano, H. (ed.) (1992). El JIT Revolución en las Fábricas, Madrid: Tecnologías de Gerencia y Producción.

Juárez Núñez, H. \& S. Babson (1999). Enfrentando el Cambio: Obreros del Automóvil y Producción Esbelta en América del Norte, 2a ed., Benemérita Universidad Autónoma de Puebla - Wayne State University Press.

Lefcovich, M. L. (2009). El Pensamiento Magro, Santa Fe: El Cid Editor.

Monden, Y. (1990). El Sistema de Producción de Toyota, Buenos Aires: Macchi.

Nakamura, M., S. Sakakibara \& R. Schroeder (1998). Adoption of Just-in-Time Manufacturing Methods at US- and Japanese-Owned Plants: Some Empirical Evidence, IEEE Trans. Engineering Management, Vol. 45 , No. 3 , pp. $230-240$.

New, S. J. (2007). Celebrating the Enigma: The Continuing Puzzle of the Toyota Production System, International Journal of Production Research, Vol. 45, No. 16, pp. 3545-3554.

Schonberger, R. J. (1987). World Class Manufacturing Casebook: Implementing JIT and TQC, Nueva York: Free Press.

Schonberger, R. J. (1996). Técnicas Japonesas de Fabricación, México: Limusa Noriega.

Sipper, D. \& R. L. Bulfin Jr. (1998). Planeación y Control de la Producción, México: McGraw-Hill.

Suzaki, K. (1993). Competitividad en Fabricación: Técnicas para la Mejora Continua, 3a ed., Madrid: TGP Hoshin. 
Ulgen, O. \& A. Gunal (1998). Simulation in the Automobile Industry, en Handbook of Simulation, J. Banks (ed.), Nueva York: Wiley, pp. 547-570.

Villaseñor Contreras, A. \& E. Galindo Cota (2008). Conceptos y Reglas de Lean Manufacturing, 2a ed., México: Limusa-ITESM.

Womack, J. P., D. T. Jones \& D. Ross (1991). The Machine That Changed the World: The Story of Lean Production, Nueva York: Harper Perennial.

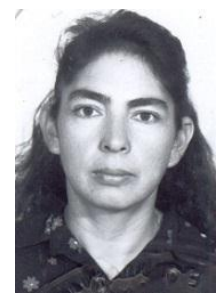

Yolanda Juárez López se graduó de Ingeniero Industrial Mecánico en el Instituto Tecnológico de Pachuca, México, y Maestría en Ingeniería en Sistemas en el Instituto Politécnico Nacional. Es alumna del Doctorado en Ciencias en Ingeniería Industrial en la Universidad Autónoma del Estado de Hidalgo, México.

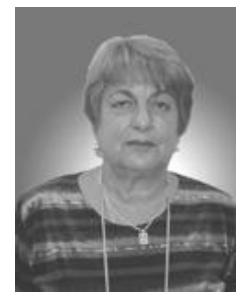

Aurora Pérez Rojas se graduó de Ingeniero Industrial, Maestría en Sistemas Automatizados de Dirección y Doctor en Ingeniería, en el Instituto Superior Politécnico José A. Echevarría, La Habana, Cuba. Es profesor investigador titular en la Universidad Autónoma del Estado de Hidalgo, México.

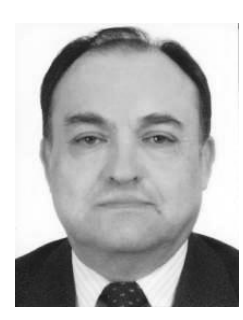

Jorge Rojas Ramírez se graduó de Ingeniero en Comunicaciones y Electrónica y de Maestro en Ingeniería Industrial en el Instituto Politécnico Nacional, México, así como de Doctorado en Automatización con Mención Honorífica en la Université de Valenciennes, Francia. Es profesor investigador en el propio Instituto Politécnico Nacional de México. 